\title{
PHOTOLITHOGRAPHY-FREE LASER-PATTERNED HF ACID-RESISTANT CHROMIUM-POLYIMIDE MASK FOR RAPID FABRICATION OF MICROFLUIDIC SYSTEMS IN GLASS
}

\author{
Konstantin O. Zamuruyev, Yuriy Zrodnikov, and Cristina E. Davis* \\ University of California, Davis, CA, USA
}

\begin{abstract}
A photolithography-free laser-patterned hydrofluoric acidresistant chromium-polyimide tape masking method for rapid prototyping of microfluidic systems in glass is presented. The patterns are defined with a diode-pumped solid-state laser. Minimum feature size is limited to $30 \mu \mathrm{m}$; minimum spacing between features is limited to $40 \mu \mathrm{m}$. The patterned glass substrates are etched in $49 \%$ hydrofluoric acid at ambient temperature with soft agitation (up to $18 \mathrm{~min}$ ). This method demonstrates comparable results to current more sophisticated masking methods in terms of the etched depth (up to $95 \mu \mathrm{m}$ in borosilicate glass), feature undercut in isotropic etch (about 1.47), mask hole density (negligible, practically zero) as well as high yield and reliability.
\end{abstract}

\section{INTRODUCTION}

The physical and chemical properties of glass as well as its compatibility with common microfabrication processes make it an advantageous material for numerous MEMS and bio-chip applications: microfluidic devices used for bioanalysis, mass spectrometry $[1,2]$, microflow cells for single molecule handling of DNA [3], micro polymerase chain reaction (PCR) devices for DNA amplification [4].

Wet etching of glass in hydrofluoric (HF) acid solution is often the preferred microfabrication method due to the high etch rate, smoothness of the etched surface, and compatibility with batch processes. Currently, there are three major masking methods used for wet etching of glass in HF acid: metal or silicon-based hard masks, photoresist masks, and using a composition of a hard mask layer with photoresist layer on top of it.

Although metal-based or silicon-based hard masks show excellent adhesion with the glass substrate and high resistance to HF acid solution [5-8], high concentration of pinholes in the field and notch defects on the edge of the etched geometries are the primary reasons for failure [9]. Increasing the thickness of the hard mask helps to reduce the number of pinhole defects but introduces the problem of residual stress in the mask [8, 10].

The use of a hard mask with a hard-baked photoresist layer on top of it has been demonstrated to be a more successful masking method for deep wet etching of glass and fused silica substrates [11-13]; reported to withstand a $20 \mathrm{~h}$ etch with resulting depth of $525 \mu \mathrm{m}$ in fused silica [14].

The application of photoresist mask alone on glass or fused silica surface was reported to survive $\sim 30$ min HF acid etch with resulting depths of $35 \mu \mathrm{m}$ in fused silica $[15,16]$. Photoresist mask preparation is sophisticated and prone to failures $[5,6]$. Thus, a combinational mask consisting of a hard-mask layer and a thick photoresist layer is the preferred masking method due to difficulties of the single-layer masks.

We report a new glass masking method with a chromium metal layer and adhesive polyimide tape (Kapton ${ }^{\circledR}$ tape) for rapid prototyping of microfluidic systems in glass using wet etching. Several examples of fabricated microsystems are demonstrated (Fig. 1). A variety of shapes and sizes are available for liquid and gas phase chemical detection applications. Successful bonding of the fabricated chips with either anodic or thermal fusion bonding demonstrates that no material was etched in the mask protected regions. The ink-filled microchannels (Fig. 1C) show no leakage or channel clogging defects. The proposed method can be applied to individual microchip-sized glass substrate or to wafer-level microfabrication processes. A 4 inch wafer containing 6 microfluidic chips is laser-patterned in $22 \mathrm{~min}$, etched for $15 \mathrm{~min}$ in $49 \% \mathrm{HF}$ acid, and anodically bonded to silicon wafer in $3 \mathrm{~h}$. The bonded wafers containing microfluidic systems are cut into individual chips with a dicing saw (Disco DAD 321, Japan).

\section{METHODS}

\section{Glass substrates and mask preparation}

The experiments are performed with 50x50x0.8 mm borosilicate (Borofloat ${ }^{\circledR} 33$ by Schott Co.) glass slides. The slides are cleaned in 4:1 Piranha solution $\left(\mathrm{H}_{2} \mathrm{SO}_{4} / \mathrm{H}_{2} \mathrm{O}_{2}\right)$ and sputtercoated with a $180 \pm 28 \mathrm{~nm}$ thick chromium layer. A $50 \mu \mathrm{m}$ thick polyimide tape with adhesive side $\left(\right.$ Kapton $^{\mathbb{B}}$ ) is applied on the chrome coated surface of the slide. The tape is gradually applied
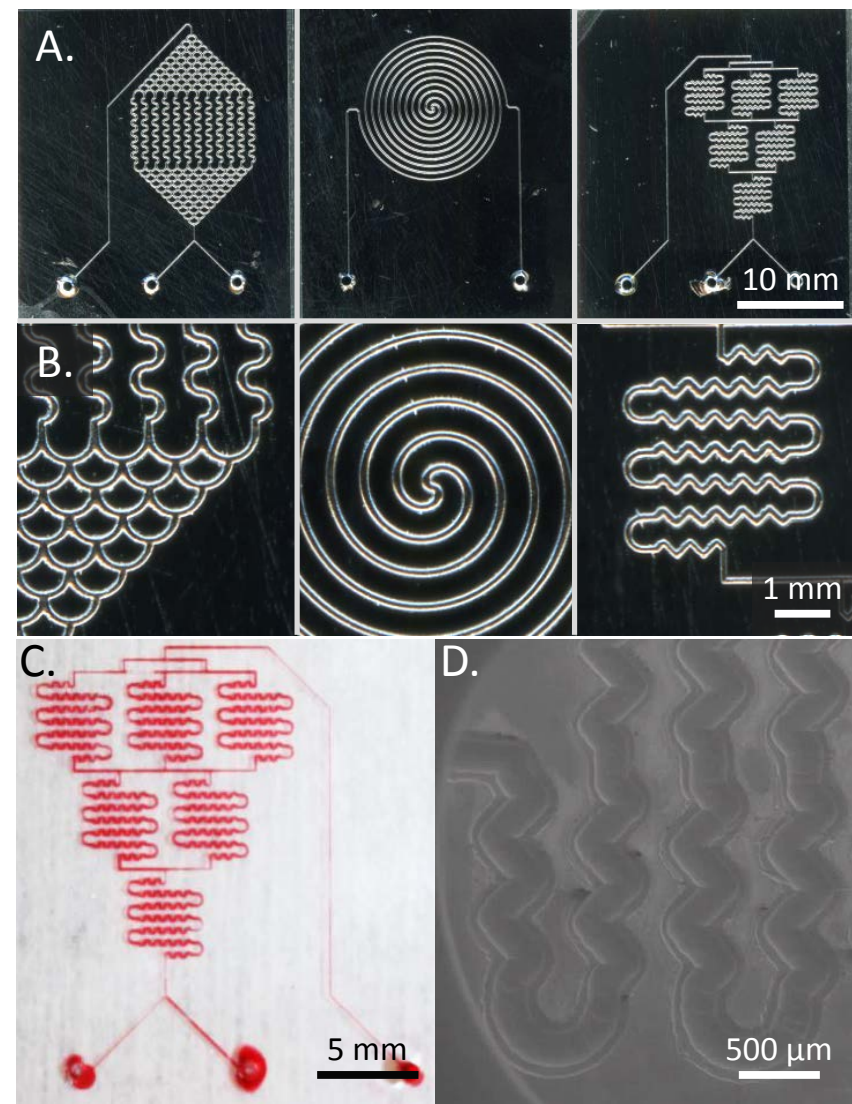

Figure 1. Microdevices with initial channel width of $40 \mu \mathrm{m}$ are etched in 49\% HF for 15 min with chromium-polyimide tape mask. Etched microchannels are $75 \mu \mathrm{m}$ deep and $225 \mu \mathrm{m}$ wide. $(A, B)$ Etched glass wafer is anodically bonded to silicon surface. (C) Surface with etched microchannels is bonded to a plain glass surface in thermal fusion. The wavy microchannels are filled with red ink. (D) SEM image of the wiggly channel $70 \mu \mathrm{m}$ deep.
Solid-State Sensors, Actuators and Microsystems Workshop Hilton Head Island, South Carolina, June 5-9, 2016 
from one side of the slide with soft plastic spatula (12.5 mm wide) to avoid air bubbles and ensure good contact of adhesive. In order to see the effect of each masking material individually, three groups of substrates are prepared: 8 glass slides with chromium metallized layer only - no adhesive polyimide tape; 6 glass slides with adhesive polyimide tape only - no chromium metal layer; 18 glass slides with chromium metal layer and adhesive polyimide tape.

\section{Defining micropatterns with laser}

The geometries are patterned with a commercial diodepumped solid-state laser (Samurai UV Marking/Micromachining System, model 3530-30; equipped with telecentric lens, $F=103$ $\mathrm{mm}$; DPSS Lasers, Santa Clara USA). The laser parameters are set at 1.5 Watt, $70 \mathrm{kHz}$ frequency, and $100 \mathrm{~mm} / \mathrm{sec}$ scan speed with path repetition of 100 . With these power parameters and installed optical lens, the minimum feature size is limited to the diameter of the laser beam $(30 \mu \mathrm{m})$. The minimum spacing between features is limited by the thermal shrinkage and adhesive contact area of the Kapton ${ }^{\circledR}$ tape to $40 \mu \mathrm{m}$. The inner area of the geometries with dimensions larger than the laser beam diameter is filled with multiple laser passes with a $10 \mu \mathrm{m}$ spacing increment from passto-pass. The minimum power input to define geometries is preferred to avoid distortion of fine features and glass cracking.

\section{Mask for etch optimization}

The mask for etch optimization consists of 12 regions containing straight lines and some chess-style squares. Each region contains 19 lines of the same width but distributed spacing. The width of the lines increases from $35 \mu \mathrm{m}$, in region one, to 200 $\mu \mathrm{m}$, in region twelve, which allows to investigate the dependence of the etch rate on geometry opening width. In each region, the spacing width between lines increases from $40 \mu \mathrm{m}$ to $373 \mu \mathrm{m}$. The variable spacing allows us to determine the minimum features spacing (highest density) as a function of the masking material and etched depth. The mask pattern is composed in CAD and imported into WinLase ${ }^{\circledR}$ commercial laser marking software (Lanmark Controls Inc., USA). The actual mask dimensions are confirmed for each masking method with a profilometer (DektakXT ${ }^{\mathrm{TM}}$, Bruker Corp.).

\section{Glass etching in 49\% HF acid}

The laser-patterned substrates are cleaned from debris with a simple DI water rinse and $1 \mathrm{~min}$ DI water sonication bath. The prepared substrates are etched in $49 \% \mathrm{HF}$ acid with soft agitation. A preliminary experiment was done before the main study to determine the maximum etch time period for each masking method. The number of prepared glass substrates in each group corresponds to the maximum number of minutes that each mask survives.

To determine the etch rate and confirm the maximum time period for each of the three masking methods, the glass substrates of one group are placed into the acid bath and taken out at one min increments until noticeable defects occur in the mask. All etching is done in a fume hood equipped for acid wet etching with an operator wearing acid resistant personal chemical protection.

\section{Characterization of the etched microstructures}

The quality of the etched features is characterized with a profilometer (DektakXT TM, Bruker Corp.). For each masking method, three scans are made at different positions across each region for every etch period ( $\mathrm{min}$ ). The measured depths of the etched lines are used to evaluate the etch rate and its dependence on mask opening width. The measured widths of the etched lines are used to evaluate the undercut ratio and measured widths of the spacing regions are used to evaluate the minimum features spacing. All profilometer data files are saved as a .CSV file for the subsequent data processing steps. The widths and depths of the etched features are analyzed with MATLAB ${ }^{\circledR}$. In each region, three profilometer scans, over 19 etched features, yield 57 profiles for evaluating depth and etch rate, width and undercut ratio per each time period (min). The only exception is the adhesive polyimide mask which shows strong dependence of undercut ratio on features spacing; only three feature profiles are available for evaluating etch parameters for each line width and spacing width.

The computer processed results are confirmed with scanning electron microscope images and measurements.

\section{RESULTS AND DISCUSSION}

In order to study the individual effect of each masking material, the three groups of substrates were compared in terms of etch duration (maximum depth) and undercut ratio.

Figure 2 shows an SEM image of the cross section of the etched profile and compares the development of the undercut distance for the masking methods. Chromium metal hard mask demonstrates minimum undercut for depths up to $45 \mu \mathrm{m}$ but fails a longer acid etch. Addition of adhesive polyimide mask over the chromium layer allows for a deeper etch with no compromise in undercut ratio. Although adhesive polyimide tape mask on glass is suitable for shallow etches, the wide undercut makes it unsuitable for applications with high density features.

Figure $3 \mathrm{~A}$ demonstrates the dependence of the etch rate on the initial mask opening width of the feature. Wider geometries are etched at faster rates. The chromium metal hard mask demonstrates little dependence of the etch rate on the mask opening width while an adhesive polyimide tape mask shows

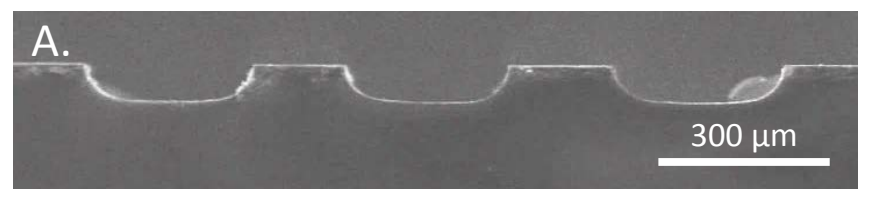

B. Cross section of the etched profile, width $(\mu \mathrm{m})$

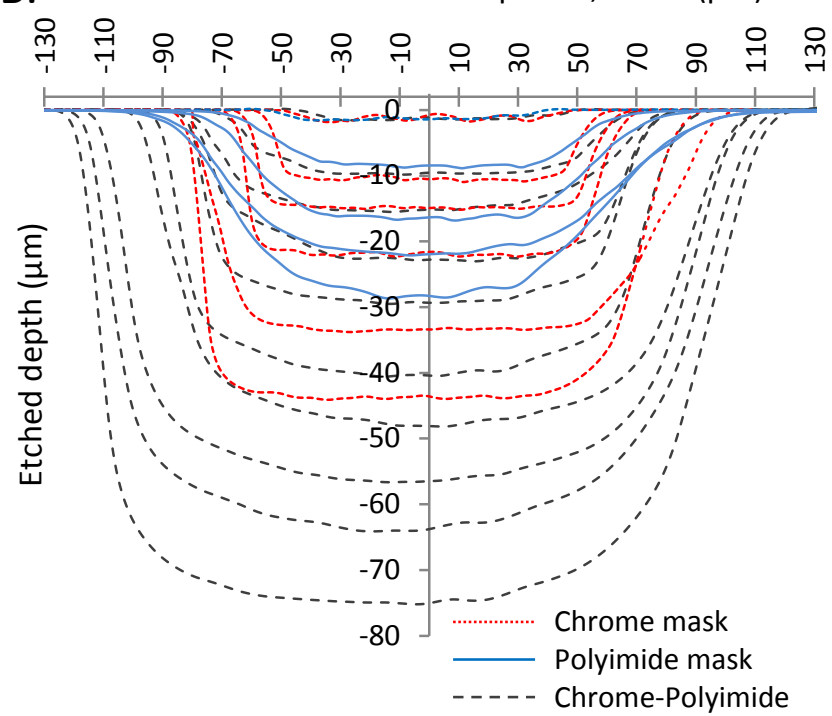

Figure 2. (A) Cross sectional profile of $75 \mu \mathrm{m}$ deep lines etched in 49\% HF using Chromium-Polyimide mask. (B) Mask undercut for the three masking methods. Initial mask opening width is $98 \mu \mathrm{m}$. The feature width increases with the etched depth. The best mask demonstrates minimal isotropic undercut and largest depth. 

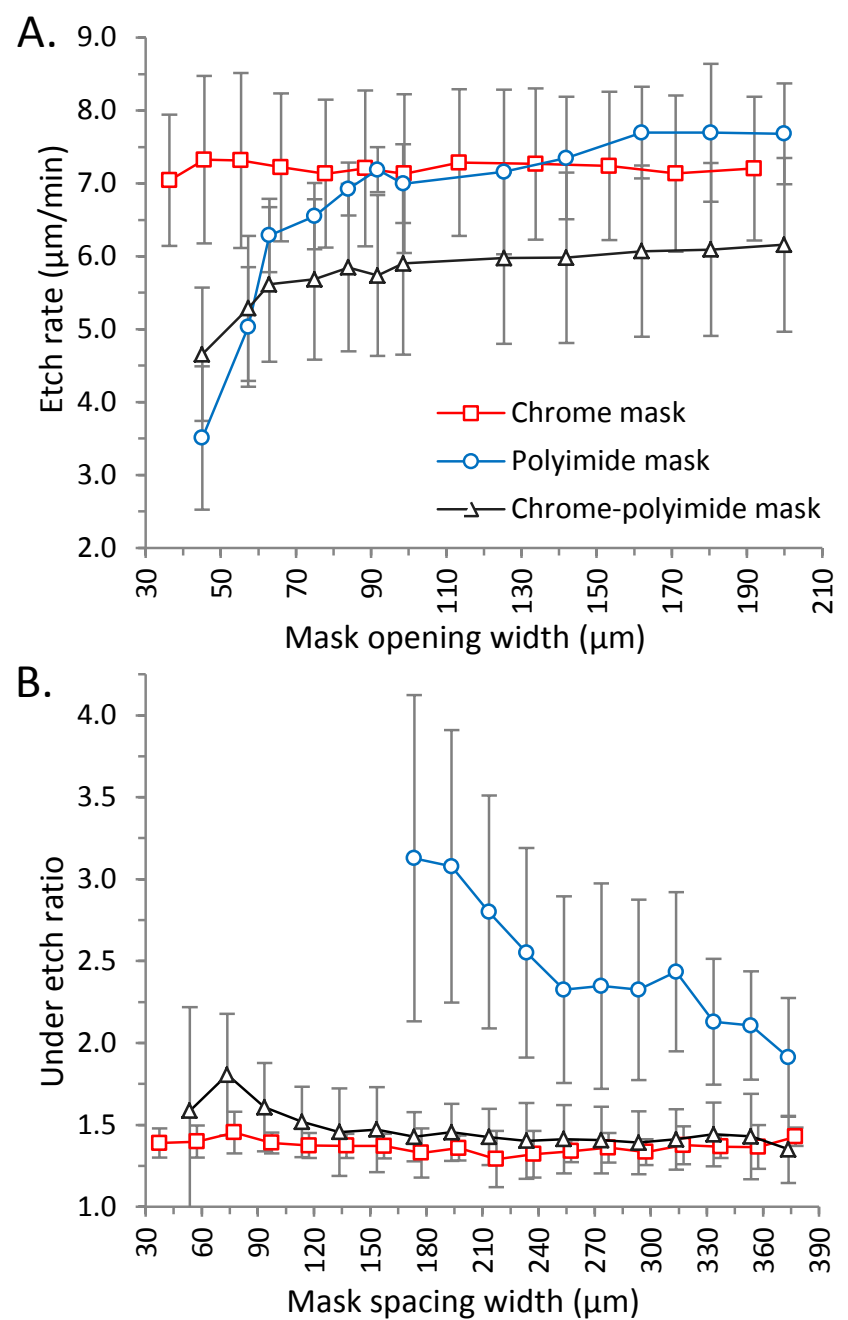

Figure 3. All values and error bars are average estimates based on 57 profilometer measurements of the etched features. Error bars show one +/- STD error. (A) Etch rate as a function of mask opening (geometry width). (B) Under etch (undercut) ratio as a function of mask spacing (width between etched regions).

strong dependence for microgeometries with mask opening width $<60 \mu \mathrm{m}$. The strong dependence of the etch rate for adhesive polyimide mask may be due to the presence of residual adhesive layer (glue) between glass and polyimide. The laser ablated parts of the glue (burned flake particles) may fill the narrow gaps and prevent acid access to the etched cavity. Longer DI water sonication bath or more optimized laser settings may reduce this dependence and allow narrow geometries to be etched to the same depth as wider openings.

Although the composite chromium-polyimide mask has the lowest etch rate, it survives the longest HF acid etch, up to $18 \mathrm{~min}$ in comparison to $6 \mathrm{~min}$ for chromium and $4 \mathrm{~min}$ for the polyimide mask. The longer etch allows us to make microstructures with twice the depth. The overall decrease in the etch rate for the chromium-polyimide tape mask in comparison to the chromium mask may be due to the increased thickness of the mask $(50 \mu \mathrm{m})$ and not fully optimized laser settings. The laser settings are kept constant for all 3 masking methods for direct comparison of mask stability in etch (eliminate dependence on laser patterning) otherwise the laser settings should be optimized for a specific masking material.
Figure 3B compares the three masking methods in terms of the geometry undercut ratio and helps to evaluate how close one can place microstructures that are to be etched to a certain depth. The isotropic undercut ratio is evaluated with measurements of width and depth of the etched features. The ratio is defined as the half difference between final width of the etched feature and initial width of the mask opening divided by the depth of the etched feature. Chromium and chromium-polyimide masking methods demonstrate a low undercut ratio $(\sim 1.47)$ and allow for a relatively high density of etched features. The adhesive polyimide tape mask demonstrates a high dependence of undercut ratio on the feature spacing. Any features that are placed closer to each other than 180 $\mu \mathrm{m}$ fail to survive a $4 \mathrm{~min}$ ( $28 \mu \mathrm{m}$ deep) etch; the mask is lifted up and peels off. The undercut ratio decreases as the spacing between features increases. The error bars in figure 3 correspond to one standard deviation above and one standard deviation below the average value.

Figure 4 compares the quality of the etched features for the 3 masking methods. The SEM images show not only undercut of the etched features but pinhole and edge defects of the mask. The chromium hard mask (Fig. 4A and B) shows a high number of pinhole defects. The defects are especially harmful when located at the edges of the etched features. The chromium mask is also very susceptible to scratches introduced while handling the substrate. The adhesive polyimide mask is simple to apply but shows severely washed away feature edges that lack sharp corners (Fig. 4C and D). The polyimide tape mask applied on a clean glass surface can withstand only a shallow etch, up to $30 \mu \mathrm{m}$ versus 45 $\mu \mathrm{m}$ for the chromium mask, and $80 \mu \mathrm{m}$ for the chromiumpolyimide mask. The chromium-polyimide mask (Fig. 4E and F) demonstrates not only the deepest etch but also the highest quality against pinhole and scratch defects. Comparing pinhole density of the 3 masks, we find that polyimide covered substrates have no pinhole defects.

The quality of the chromium layer for all chromium layer masked and chromium-polyimide masked substrates should be the same because they all were coated in a single batch. Thus better optimization for quality of the chromium layer will improve both masking methods.

The presented results characterize masking methods for the deepest (aggressive) etch in 49\% HF acid. Shallow and more controlled etches can be done with various concentrations of Buffered Oxide Etchant (BOE) solutions. The proposed mask can withstand an hours-long BOE etch but the etched depth is very shallow due to lower etch rate of glass. The mask eventually fails mainly due to duration of the etch period.

\section{CONCLUSION}

We describe a new glass masking method with: mask stability in terms of maximum etch rate and maximum etched depth; feature density in terms of undercut ratio and minimum feature spacing. Due to its resolution and etch depth limitations, the proposed method is not suggested as a full substitute to currently-used methods involving a metal hard mask with thick photoresist. But we find the proposed method to be very useful for rapid microfabrication of gas and liquid microfluidic systems because of its simplicity and reliability. The demonstrated 6 microfluidic chips, on a single 4 inch wafer, were fabricated in $1 \mathrm{~h}$ and anodically bonded in $3 \mathrm{~h}$ while preparation of a reliable metalphotoresist mask requires hours of cleanroom time. Air bubblefree application of polyimide tape mask on chromium coated glass surface demonstrated repeatable results with multiple wafers. The use of a laser to define the microfluidic features in the chromiumpolyimide tape mask side-steps the costly and time consuming 


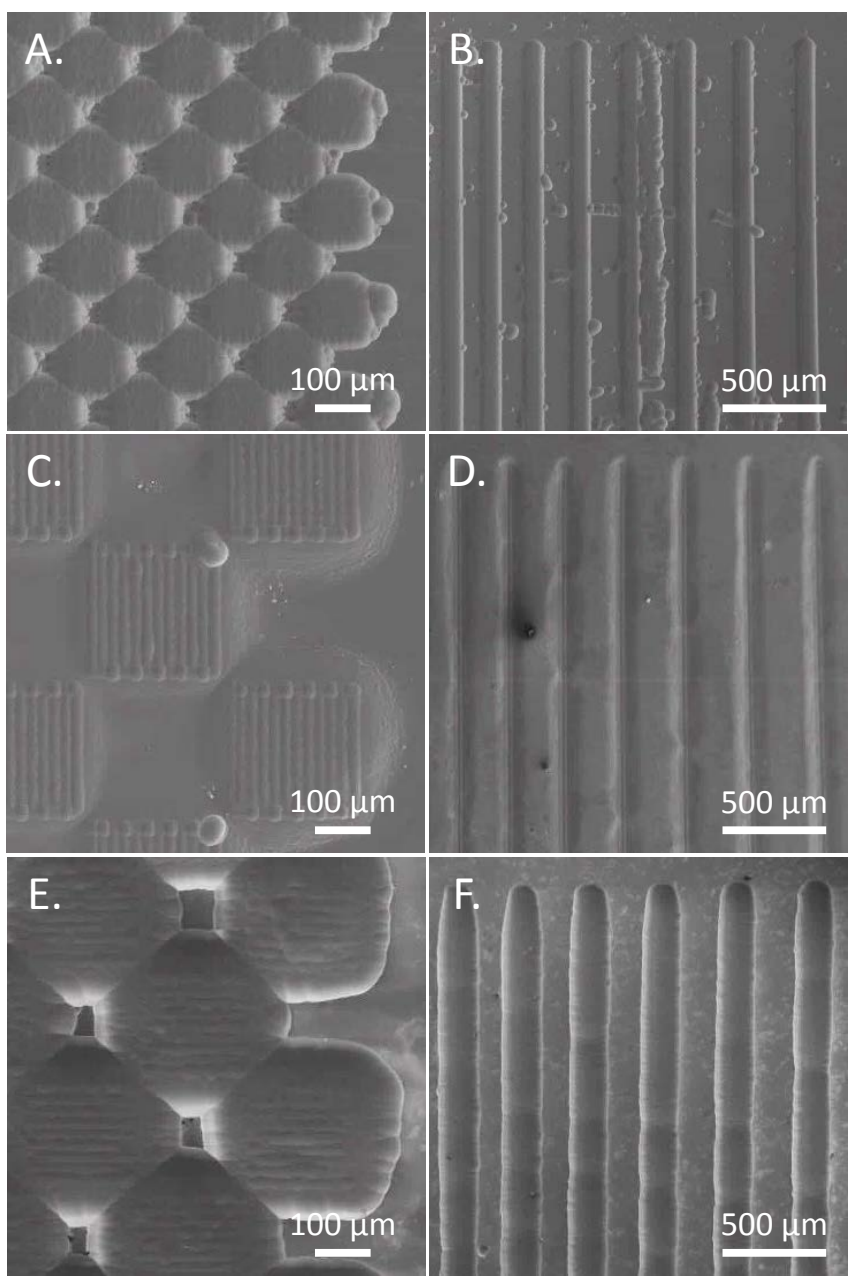

Figure 4. Compare the quality of the etched features for the three masking methods. (A, B) Chromium mask; 30 m deep etch. Initial square size $90 \mu \mathrm{m}$ and line width $45 \mu \mathrm{m}$. $(C, D)$ Polyimide tape mask; $17 \mu \mathrm{m}$ deep etch. Initial square size $250 \mu \mathrm{m}$ and line width $57 \mu \mathrm{m}$. (E, F) Chromium and polyimide tape mask; $65 \mu \mathrm{m}$ deep etch. Initial square size $200 \mu \mathrm{m}$ and line width $57 \mu \mathrm{m}$.

photolithography step. Possible rapid change of microfeatures layout and size is very beneficial for faster microfluidic chip design development and performance optimization.

The proposed method of fabrication can potentially be implemented in research institutions with no access to a standard clean-room facility. Chromium coated glass slides can be acquired from commercial supplier. The laser patterning step does not require a dust-free environment. Glass HF etch can be done in a regular fume hood equipped for wet etching. The bonding step is the only step that requires a particle free environment.

\section{REFERENCES}

[1] Koh, Y., et al., Bead affinity chromatography in a temperature-controllable microsystem for biomarker detection. Analytical and Bioanalytical Chemistry, 2012. 404(8): p. 2267-2275.

[2] Saarela, V., et al., Glass microfabricated nebulizer chip for mass spectrometry. Lab on a Chip, 2007. 7(5): p. 644-646.

[3] Rusu, C., et al., Direct integration of micromachined pipettes in a flow channel for single DNA molecule study by optical tweezers. Journal of Microelectromechanical Systems, 2001. 10(2): p. 238-246.
[4] Obeid, P.J., et al., Microfabricated device for DNA and RNA amplification by continuous-flow polymerase chain reaction and reverse transcription-polymerase chain reaction with cycle number selection. Analytical Chemistry, 2003. 75(2): p. 288-295.

[5] Zhu, H.X., et al., Characterization of deep wet etching of fused silica glass for single cell and optical sensor deposition. Journal of Micromechanics and Microengineering, 2009. 19(6).

[6] Bien, D.C.S., et al., Characterization of masking materials for deep glass micromachining. Journal of Micromechanics and Microengineering, 2003. 13(4): p. S34-S40.

[7] Steingoetter, I. and H. Fouckhardt, Deep fused silica wet etching using an Au-free and stress-reduced sputter-deposited $\mathrm{Cr}$ hard mask. Journal of Micromechanics and Microengineering, 2005. 15(11): p. 2130-2135.

[8] Ong, Y.Y., et al., Process Analysis and Optimization on PECVD Amorphous Silicon on Glass Substrate. International Mems Conference 2006, 2006. 34: p. 812-817.

[9] Simpson, P.C., A.T. Woolley, and R.A. Mathies, Microfabrication Technology for the Production of Capillary Array Electrophoresis Chips. Biomedical Microdevices, 1998. 1(1): p. 7-25.

[10] Iliescu, C., J.M. Miao, and F.E.H. Tay, Stress control in masking layers for deep wet micromachining of Pyrex glass. Sensors and Actuators a-Physical, 2005. 117(2): p. 286-292.

[11] Bu, M.Q., et al., A new masking technology for deep glass etching and its microfluidic application. Sensors and Actuators a-Physical, 2004. 115(2-3): p. 476-482.

[12] Iliescu, C., F.E.H. Tay, and J.M. Miao, Strategies in deep wet etching of Pyrex glass. Sensors and Actuators a-Physical, 2007. 133(2): p. 395-400.

[13] Ceyssens, F. and R. Puers, Deep etching of glass wafers using sputtered molybdenum masks. Journal of Micromechanics and Microengineering, 2009. 19(6).

[14] Jin, J.Y., et al., Deep wet etching of borosilicate glass and fused silica with dehydrated AZ4330 and a Cr/Au mask. Journal of Micromechanics and Microengineering, 2014. 24(1).

[15] Grosse, A., M. Grewe, and H. Fouckhardt, Deep wet etching of fused silica glass for hollow capillary optical leaky waveguides in microfluidic devices. Journal of Micromechanics and Microengineering, 2001. 11(3): p. $257-$ 262.

[16] Lin, C.H., et al., A fast prototyping process for fabrication of microfluidic systems on soda-lime glass. Journal of Micromechanics and Microengineering, 2001. 11(6): p. 726732 .

\section{CONTACT}

*Cristina Davis, cedavis@ucdavis.edu

\section{ACKNOWLEDGEMENTS}

Partial support was provided by: the NIH National Center for Advancing Translational Sciences (NCATS) through grant \#UL1 TR000002 [CED]; NIH award U01 EB0220003-01 [CED]; NIH award 1P30ES023513-01A1 [CED]; and NSF award \#1255915. Student support was provided by NIH award T32 HL07013 [KOZ] and NIH award \# P42ES004699 [KOZ]. The contents of this manuscript are solely the responsibility of the authors and do not necessarily represent the official views of the funding agencies.

The authors are grateful to lab members Sierra Spitulski and Daniel Peirano for assistance with computerized processing of CAD and profilometer data files. 